\title{
Automata-Based Optimization of Interaction Protocols for Scalable Multicore Platforms
}

\author{
Sung-Shik T.Q. Jongmans, Sean Halle, and Farhad Arbab \\ Centrum Wiskunde \& Informatica, Amsterdam, The Netherlands \\ $\{$ jongmans, sean, farhad\}@cwi.nl
}

\begin{abstract}
Multicore platforms offer the opportunity for utilizing massively parallel resources. However, programming them is challenging. We need good compilers that optimize commonly occurring synchronization/ interaction patterns. To facilitate optimization, a programming language must convey what needs to be done in a form that leaves a considerably large decision space on how to do it for the compiler/run-time system.

Reo is a coordination-inspired model of concurrency that allows compositional construction of interaction protocols as declarative specifications. This form of protocol programming specifies only what needs to be done and leaves virtually all how-decisions involved in obtaining a concrete implementation for the compiler and the run-time system to make, thereby maximizing the potential opportunities for optimization. In contrast, the imperative form of protocol specification in conventional concurrent programming languages, generally, restrict implementation choices (and thereby hamper optimization) due to overspecification.

In this paper, we use the Constraint Automata semantics of Reo protocols as the formal basis for our optimizations. We optimize a generalization of the producer-consumer pattern, by applying CA transformations and prove the correctness of the transforms.
\end{abstract}

\section{Introduction}

Context. Coordination languages have emerged for the implementation of protocols among concurrent entities (e.g., threads on multicore hardware). One such language is Reo [12, a graphical language for compositional construction of connectors (i.e., custom synchronization protocols). Figure 1a shows an example. Briefly, a connector consists of one or more edges (henceforth referred to as channels), through which data items flow, and a number of nodes (henceforth referred to as ports), on which channel ends coincide. The connector in Figure 1a contains three different channel classes, including standard synchronous channels (normal arrows) and asynchronous channels with a buffer of capacity 1 (arrows decorated with a white rectangle, which represents a buffer). Through connector composition (the act of gluing connectors together on their shared ports), programmers can construct arbitrarily complex connectors. As Reo supports both synchronous and asynchronous channels, connector composition enables mixing synchronous and asynchronous communication within the same specification. 


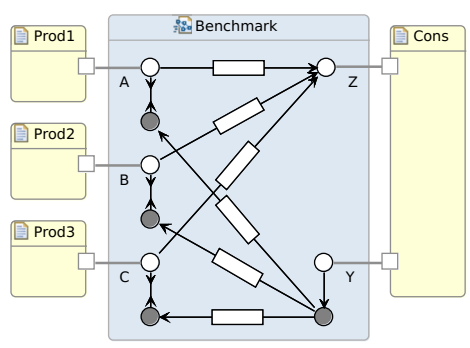

(a) Connector

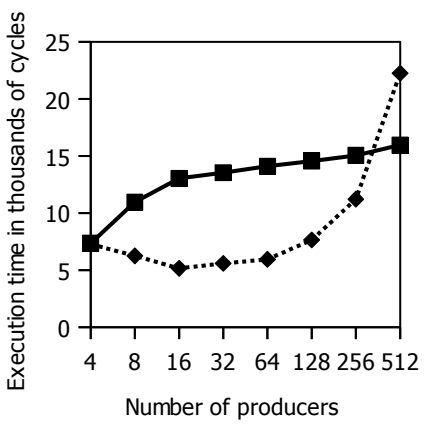

(b) Per-interaction overhead for the Pthreads-based implementation (continuous line; squares) and the pre-optimized CA-based implementation (dotted line; diamonds)

Fig. 1. Producers-consumer benchmark

Especially when it comes to multicore programming, Reo has a number of advantages over conventional programming languages with a fixed set of low-level synchronization constructs (locks, mutexes, etc.). Programmers using such a conventional language have to translate the synchronization needs of their protocols into the synchronization constructs of that language. Because this translation occurs in the mind of the programmer, invariably some context information either gets irretrievably lost or becomes implicit and difficult to extract in the resulting code. In contrast, Reo allows programmers to compose their own synchronization constructs (i.e., connectors) at a high abstraction level to perfectly fit the protocols of their application. Not only does this reduce the conceptual gap for programmers, which makes it easier to implement and reason about protocols, but by preserving all relevant context information, such user-defined synchronization constructs also offer considerable novel opportunities for compilers to do optimizations on multicore hardware. This paper shows one such occasion.

Additionally, Reo has several software engineering advantages as a domainspecific language for protocols 3 . For instance, Reo forces developers to separate their computation code from their protocol code. Such a separation facilitates verbatim reuse, independent modification, and compositional construction of protocol implementations (i.e., connectors) in a straightforward way. Moreover, Reo has a strong mathematical foundation [4, which enables formal connector analyses (e.g., deadlock detection, model checking [5]).

To use connectors in real programs, developers need tools that automatically generate executable code for connectors. In previous work [6], we therefore developed a Reo-to-C compiler, based on Reo's formal semantics of constraint 
automata (CA) [7. In its simplest form, this tool works roughly as follows. First, it extracts from an input XML representation of a connector a list of its primitive constituents 1 Second, it consults a database to find for every constituent in the list a "small" CA that formally describes the behavior of that particular constituent. Third, it computes the product of the CA in the constructed collection to obtain one "big" CA describing the behavior of the whole connector. Fourth, it feeds a data structure representing that big CA to a template. Essentially, this template is an incomplete C file with "holes" that need be "filled". The generated code simulates the big CA by repeatedly computing and firing eligible transitions in an event-driven fashion. It runs on top of Proto-Runtime [89], an execution environment for $\mathrm{C}$ code on multicore hardware. A key feature of ProtoRuntime is that it provides more direct access to processor cores and control over scheduling than threading libraries based on os threads, such as Pthreads [10.

Problem. Figure 1a shows a connector for a protocol among $k=3$ producers and one consumer in a producers-consumer benchmark. Every producer loops through the following steps: (i) it produces, (ii) it blocks until the consumer has signaled ready for processing the next batch of productions, and (iii) it sends its production. Meanwhile, the consumer runs the following loop: (i) it signals ready, and (ii) it receives exactly one production from every producer in arbitrary order. We compared the CA-based implementation generated by our tool with a hand-crafted implementation written by a competent $\mathrm{C}$ programmer using Pthreads, investigating the time required for communicating a production from a producer to the consumer as a function of the number of producers.

Figure $1 \mathrm{~b}$ shows our results. On the positive side, for $k \leq 256$, the CA-based implementation outperforms the hand-crafted implementation. For $k=512$, however, the Pthreads-based implementation outperforms the generated implementation. Moreover, the dotted curve looks disturbing, because it grows morethan-linearly in $k$ : indeed, the CA-based implementation scales poorly. (We skip many details of this benchmark, including those of the Pthreads-based implementation, and the meaning/implications of these experimental results. The reason is that this paper is not about this benchmark, and its details do not matter. We use this benchmark only as a concrete case to better explain problems of our compilation approach and as a source of inspiration for solutions.)

Contribution. In this paper, we report on work at improving the scalability of code generated by our Reo-to-C compiler. First, we identify a cause of poor scalability: briefly, computing eligibility of $k$ transitions in producers-consumerstyle protocols (and those generalizations thereof that allow any synchronization involving one party from every one of $\ell$ groups) takes $\mathcal{O}(k)$ time instead of $\mathcal{O}(1)$, of which the Pthreads-based implementation shows that it is possible. Second, to familiarize the reader with certain essential concepts, we explain a manual solution (in terms of Reo's CA semantics) that achieves $\mathcal{O}(1)$. Third, we propose

${ }^{1}$ Programmers can use the ECT plugins for Eclipse (http://reo.project.cwi.nl) to draw connectors such as the one in Figure 1a, internally represented as XML. 


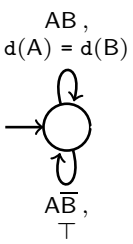

(a) LossySync
$\mathrm{AZ} \overline{\mathrm{BC}}$

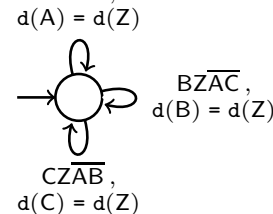

(b) Merger 3

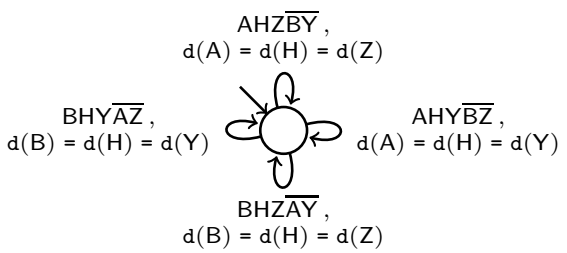

(c) Hourglass

Fig. 2. Example CA, called LossySync, Merger 3 and Hourglass

an automated, general solution, built upon the same concepts as the manual solution. We formalize this automated solution and prove it correct. Although inspired by our work on Reo and formulated in terms of $\mathrm{CA}$, we make more general contributions beyond Reo and CA, better explained in our conclusion.

We organized the rest of this paper as follows. In Section 2, we explain CA. In Section 3. we analyze how the Pthreads-based implementation avoids scalability issues and how we can export that to our setting. In Sections 46 , we automate the solution proposed in Section 3. Section 7 concludes this paper. Definitions and detailed proofs appear in the appendix of a technical report [1].

Although inspired by Reo, we can express our main results in a purely automata-theoretic setting. We therefore skip a primer on Reo [1]2 .

\section{Constraint Automata}

Constraint automata are a general formalism for describing systems behavior and have been used to model not only connectors but also, for instance, actors [12. Figure 2 shows examples 2,3 In the context of this paper, a CA specifies when during execution of a connector which data items flow where. Structurally, every CA consists of finite sets of states, transitions between states, and ports. States represent the internal configurations of a connector, while transitions describe its atomic execution steps. Every transition has a label that consists of two elements: a synchronization constraint (SC) and a data constraint (DC). An SC is a

2 The LossySync CA models a connector with one input port A and one output port B. It repeatedly chooses between two atomic execution steps (constrained by availability of pending I/O operations): synchronous flow of data from A to B or flow of data only on A (after which the data is lost, before reaching B). The Merger ${ }_{3}$ CA models a connector with three input ports $A, B$, and $C$ and one output port $Z$. It repeatedly chooses between three atomic execution steps: synchronous flow of data from $A$ to $Z$, from $B$ to $Z$, or from $C$ to $Z$. Finally, the Hourglass CA models a connector with two input ports $A$ and $B$, one internal port $H$, and two output ports $Y$ and $Z$. It repeatedly chooses between four atomic execution steps: synchronous flow of data from $A$ via $H$ to $\mathrm{Y}$, from $\mathrm{A}$ via $\mathrm{H}$ to $\mathrm{Z}$, from $\mathrm{B}$ via $\mathrm{H}$ to $\mathrm{Y}$, or from $\mathrm{B}$ via $\mathrm{H}$ to $\mathrm{Z}$.

${ }^{3}$ We show only single state CA for simplicity. Generally, a CA can have any finite number of states, and the results in this paper are applicable also to such CA. 


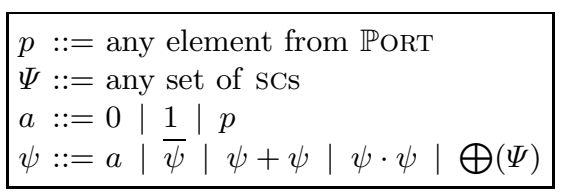

(a) Synchronization constraints

$$
\begin{array}{|l}
\hline p::=\text { any element from } \mathbb{P O R T} \\
P::=\text { any subset of } \mathbb{P} \text { ORT } \\
b::=\perp|\top| \operatorname{Eq}(P) \mid \mathrm{d}(p)=\mathrm{d}(p) \\
\phi::=b|\neg \phi| \phi \vee \phi \mid \phi \wedge \phi
\end{array}
$$

(b) Data constraints

Fig. 3. Syntax

propositional formula that specifies which ports synchronize in a firing transition (i.e., where data items flow); a DC is a propositional formula that (under)specifies which particular data items flow where. For instance, in Figure 2a, the $\mathrm{DC} d(A)=$ $d(B)$ means that the data item on $A$ equals the data item on $B$; the DC $T$ means that it does not matter which data items flow. Let PORT denote the global set of all ports. Formally, an SC is a word $\psi$ generated by the grammar in Figure $3 \mathrm{a}$ while a DC is a word $\phi$ generated by the grammar in Figure $3 \mathrm{~b}$.

Figure $3 \mathrm{a}$ generalizes the original definition of sCs as sets of ports interpreted as conjunctions [7] (shortly, we elaborate on the exact correspondence). Operator $\bigoplus$ is a uniqueness quantifier: $\bigoplus(\Psi)$ holds if exactly one sc in $\Psi$ holds. Also, we remark that predicate $\mathrm{Eq}(P)$ is novel. It holds if equal data items are distributed over all ports in $P$. In many practical cases - but not all-we can replace a DC of the shape $\mathrm{d}\left(p_{1}\right)=\mathrm{d}\left(p_{2}\right)$ with $\operatorname{Eq}(P)$ if $\left\{p_{1}, p_{2}\right\} \subseteq P$. In the development of our optimization technique, $\mathrm{Eq}(P)$ plays an important role (see also Section 7).

Let DATA denote the set of all data items. Formally, we interpret sCs and DCs over distributions of data over ports, $\delta:$ PORT $\rightarrow$ DATA, using relations $\stackrel{s c}{=}$ and $\stackrel{\mathrm{dc}}{=}$ and the corresponding equivalence relations $\equiv_{\mathrm{sc}}$ and $\equiv_{\mathrm{dc}}$. Their definition for negation, disjunction, and conjunction is standard; for atoms, we have:

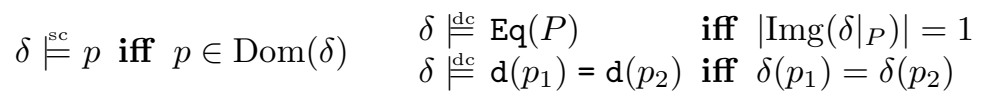

Let $\sum\left(\left\{\psi_{1}, \ldots, \psi_{k}\right\}\right)$ and $\Pi\left(\left\{\psi_{1}, \ldots, \psi_{k}\right\}\right)$ abbreviate $\psi_{1}+\cdots+\psi_{k}$ and $\psi_{1} \cdots \psi_{k}$, let $\mathbb{S C}$ denote the sets of all sCs, and let $\mathrm{SC}(P)$ and $\mathrm{DC}(P)$ denote the sets of all SCs and all DCs over ports in $P$.

A constraint automaton is a tuple $(Q, P, \longrightarrow, \imath)$ with $Q$ a set of states, $P \subseteq \mathbb{P O R T}$ a set of ports, $\longrightarrow \subseteq Q \times \mathrm{SC}(P) \times \mathrm{DC}(P) \times Q$ a transition relation labeled with $[\mathrm{SC}, \mathrm{DC}]$-pairs of the form $(\psi, \phi)$, and $\imath \in Q$ an initial state.

A distribution $\delta$ represents a single atomic execution step of a connector in which data item $\delta(p)$ flows on port $p$ (for all ports in the domain of $\delta$ ). A CA $\alpha$ accepts streams (i.e., infinite sequences) of such distributions. Every such a stream represents one possible infinite execution of the connector modeled by $\alpha$. Intuitively, to see if $\alpha$ accepts a stream $\sigma$, starting from the initial state, take the first element $\sigma(0)$ from the stream, check if $\alpha$ has a $(\psi, \phi)$-labeled transition from the current state such that $\sigma(0) \stackrel{s c}{=} \psi$ and $\sigma(0) \stackrel{\text { dc }}{=} \phi$, and if so, make this transition, remove $\sigma(0)$ from the stream, and repeat. 


$$
\underset{\text { alpha }}{g_{1}} \stackrel{\alpha}{\downarrow}
$$

(a) Current

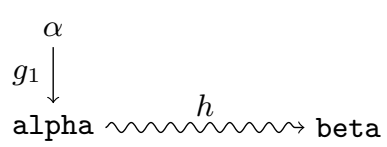

(b) Manual improvement

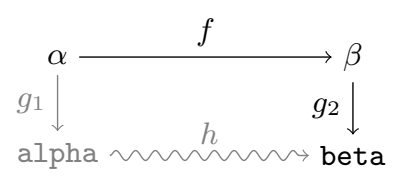

(c) Automated improvement

Fig. 4. Code generation diagrams

Our CA definition generalizes the original definition of CA 7], because Figure 3a generalizes the original definition of sCs. However, CA as originally defined still play a role in the development of our optimization technique: all input CA that this technique operates on are original. Therefore, we make more precise what "originality" means. First, let a $P$-complete product be a product of either a positive or a negative literal for every port in $P$. Intuitively, a $P$-complete product specifies not only which ports participate in a transition, but it also makes explicit which ports idle in that transition. Let $\operatorname{cp}\left(P, P_{+}\right)$denote a $P$ complete product with positive literals $P_{+} \subseteq P$. Then, we call an sC $\psi$ original if a set $P_{+}$exists such that $\operatorname{cp}\left(P, P_{+}\right) \equiv \psi$ (originally, set $P_{+}$would be the $\mathrm{SC}$ ); we call a CA original if it has only original sCs. All CA in Figure 2 are original.

We adopt bisimilarity on CA as behavioral congruence, derived from the definition for original CA of Baier et al. [7. Roughly, if $\alpha$ and $\beta$ are bisimilar, denoted as $\alpha \sim \beta, \alpha$ can simulate every transition of $\beta$ in every state and vice versa (see Definition 32 in [11, Appendix A]).

\section{Enhancing Scalability: Problem and Solution}

We study the scalability of code generated by our compiler using Figure 4 We start with Figure 4a, which summarizes the code generation process of our current tool: given an original CA $\alpha$ (computed for the connector to generate code for), it generates a piece of code alpha by applying transformation $g_{1}$.

Essentially, alpha consists of an event-driven handler, which simulates $\alpha$. This handler runs concurrently with the code of its environment (i.e., the code of the entitites under coordination), whose events (i.e., I/O operations performed on ports) it listens and responds to, as follows. Whenever the environment performs an I/O operation on a port $p$, it assigns a representation of that operation to an event variable in a data structure for $p$ (also generated by transformation $g_{1}$ and part of alpha). This causes the handler to start a new round of simulating $\alpha$. Based on the state of $\alpha$ that the handler at that point should behave as, the handler knows which transitions of $\alpha$ may fire. Which of those transitions actually can fire, however, depends also on the pending events that previously occurred (i.e., the pending I/O operations on ports). To investigate this, the handler checks for every transition that may fire if the pending events (including the new one) can constitute a distribution $\delta$ that satisfies the transition's label. If so, the handler fires the transition: it distributes data over ports according to 
$\delta$, and the events involved dissolve. Otherwise, if no transition can fire, all events remain for the next round, and the handler goes dormant.

Now, recall our producers-consumer benchmark in Section 1 Figure 2b shows the $\mathrm{CA}$ for the connector in Figure 1a 4 Generally, for an arbitrary number of producers $k$, the corresponding CA $\alpha_{k}$ has $k$ transitions. Consequently, in the worst case, the handler in the generated alpha_k code performs $k$ checks in every event handling round, which takes $\mathcal{O}(k)$ time. Figure $1 \mathrm{~b}$ shows this as a morethan-linear increase in execution time for the dotted curve 5 The Pthreads-based implementation, in contrast, uses a queue for lining up available productions. To receive a production, the consumer simply dequeues, which takes only $\mathcal{O}(1)$ time (ignoring, for simplicity, the overhead of synchronizing queue accesses). Figure 1b shows this as a linear increase in execution time for the continuous curve.

Intuitively, by checking all transitions to make the consumer receive, the generated CA-based implementation performs an exhaustive search for a particular producer that sent a production. In contrast, by using a queue, the Pthreadsbased implementation avoids such a search: the queue embodies that in this protocol, it does not matter which particular producer sent a production as long as some producer has done so (in which case the queue is nonempty). The producers are really indistinguishable from the perspective of the consumer. Thus, to improve the scalability of code generated by our tool, we want to export the idea of "using queues to leverage indistinguishability" to our setting.

Figure $4 \mathrm{~b}$ shows a first attempt at achieving this goal: we introduce a manual transformation $h$ that takes alpha as input and hacks together a new piece of code beta, which should (i) behave as alpha, (ii) demonstrate good scalability, and (iii) use queues. For instance, in our producers-consumer example $(k=3)$, $h$ works roughly as follows. First, $h$ replaces the event variable in the data structure for every port $p \in\{\mathrm{A}, \mathrm{B}, \mathrm{C}, \mathrm{Z}\}$ with an eventQueue variable that points to a queue of pending events. In this new setup, to perform an I/O operation, the environment enqueues an eventQueue, while handler code tests eventQueues for nonemptiness to check SCs, peeks eventQueues to check DCs, and dequeues eventQueues to fire transitions. Subsequently, $h$ adds initialization code to alpha to ensure that the eventQueue variables of ports $A, B$, and $C$ all point to the same shared queue, while the eventQueue variable for port $Z$ points to a different queue. Here, $h$ effectively exploits the indistinguishability property of producers

${ }^{4}$ To be precise, the $\mathrm{CA}$ in Figure $2 \mathrm{~b}$ describes the behavior of one of the synchronous regions of the connector in Figure 1a (i.e., a particular subconnector of the whole). This point is immaterial to our present discussion, however, and ignoring it simplifies our presentation without loss of generality or applicability.

5 The growth is more-than-linear instead of just linear because of the barrier in the protocol. When producer $P$ is ready to send its $(i+1)$-th production, the consumer may not yet have received the $i$-th production from all other producers. Then, $P$ must wait until the consumer signals ready (i.e., the barrier). In the worst case, however, the consumer has received an $i$-th production only from $P$ such that $P$ has to wait $(k-1) \cdot \mathcal{O}(k)$ time. Afterward, it takes another $\mathcal{O}(k)$ time for $P$ to send its $(i+1)$-th production. Consequently, sending the $(i+1)$-th production takes $k \cdot \mathcal{O}(k)$ time, and the complexity of sending a production lies between $\mathcal{O}(k)$ and $\mathcal{O}\left(k^{2}\right)$. 


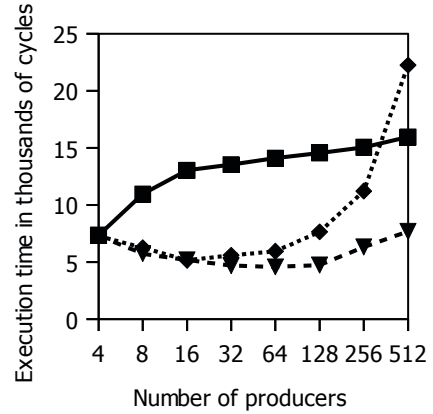

(a) With barrier

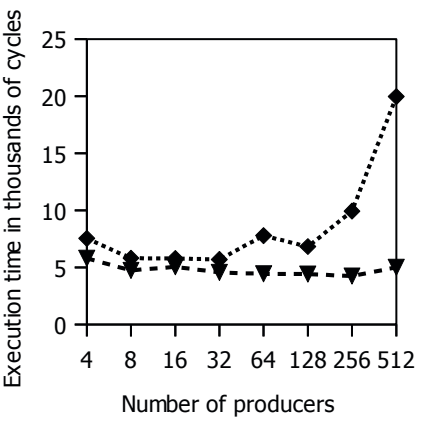

(b) Without barrier

Fig. 5. Per-interaction overhead for the Pthreads-based implementation (continuous line; squares), the pre-optimized CA-based implementation (dotted line; diamonds), and the optimized, $h$-transformed CA-based implementation (dashed line; triangles) of the producers-consumer scenario in Figure 1

by making the ports that those producers use indistinguishable in our setting. Finally, $h$ updates the handler code such that it processes the shared queue only once per event handling round instead of thrice (i.e., once for every transition). From an automata-theoretic perspective, $h$ replaces the implementation of the three "physical" transitions with an implementation of one merged "virtual" transition. When the handler fires this virtual transition at run-time, it actually fires one of the three physical transitions.

Property (iii) holds of the piece of code beta resulting from applying $h$ to alpha as just described. Figure 5 shows that also property (ii) holds. The dashed curve in Figure 5 a shows execution times of $h$-transformed code of the CA-based implementation in the producers-consumer benchmark. The $h$-transformed code scales much better than the original code. Additionally, Figure 5b shows execution times of the producers-consumer benchmark without a barrier (i.e., producers send productions whenever they want). In this variant, $h$ achieves even better results: it transforms a poorly scalable program into one that scales perfectly 6

Establishing property (i), however, is problematic. Although we can informally argue that it holds, proving this - formally showing the equivalence of two concurrent $\mathrm{C}$ programs - seems prohibitively complex. That aside, the manual nature of $h$ makes its usage generally impractical, and it seems extremely difficult to automate it: an automated version of $h$ would have to analyze $\mathrm{C}$ code

${ }^{6}$ Of course, in many cases and for many applications, a purely asynchronous producers-consumer protocol without a barrier, as in Figure 5b suffices. The reason that we initially focused on a producers-consumer protocol with a barrier, which is also useful yet in other applications, is that its mix of synchrony and asynchrony makes it a harder, and arguably more interesting, protocol to achieve good scalability for. Comparing the results in Figures $5 \mathrm{a}$ and $5 \mathrm{~b}$ also shows this. 
to recover relevant context information about the protocol, which is not only hard but often theoretically impossible. Similarly, it seems infeasible to write an optimizing compiler able to transform, for instance, less scalable Pthreads-based implementations of the producers-consumer scenario (without queues) into the Pthreads-based implementation (with queues) used in our benchmark. The inability of compilers for lower-level languages to do such optimizations seems a significant disadvantage of using such languages for multicore programming.

We therefore pursue an alternative approach, outlined in Figure 4c we introduce a transformation $f$ that takes CA $\alpha$ as input-instead of the low-level $\mathrm{C}$ code generated for it - and transforms it into an equivalent automaton $\beta$, a variant of $\alpha$ with merged transitions (cf. transformation $h$, which implicitly replaced the implementation of several physical transitions with one virtual transition). Crucially, $\alpha$ still explicitly contains all relevant context information about the protocol, exactly what makes $f$ eligible to automation. In particular, to merge transitions effectively, $f$ carefully inspects transition labels and takes port indistinguishability into account. The resulting merged transitions have an "obvious" and mechanically obtainable implementation using queues. A subsequent transformation $g_{2}$, from $\beta$ to beta, performs this final straightforward step.

We divide transformation $\alpha \stackrel{f}{\longrightarrow} \beta$ into a number of constituent transformations $\alpha \stackrel{f_{1}}{\longrightarrow} \beta^{\prime} \stackrel{f_{2}}{\longrightarrow}\left(\beta^{\prime}, \Gamma\right) \stackrel{f_{3}}{\longrightarrow} \beta$, discussed in detail in the following sections.

\section{Transformation $f_{1}$ : Preprocessing}

Transformation $f_{1}$ aims at merging transitions $t_{1}, \ldots, t_{k}$ into one transition $(q$, $\left.\psi, \mathrm{Eq}(P), q^{\prime}\right)$, where $\psi=\sum\left(\left\{\psi_{1}, \ldots, \psi_{k}\right\}\right)$. It consists of two steps.

In the first step, transformation $f_{1}$ replaces DCs on transitions of $\alpha=(Q$, $P, \longrightarrow, \imath$ ) with $\mathrm{Eq}(P)$, as follows. Because $\alpha$ is an original CA (our current code generator can handle only original CA), every SC in $\alpha$ is an original SC: for every transition label $(\psi, \phi)$, a set of ports $P_{+}$exists such that $\operatorname{cp}\left(P, P_{+}\right) \equiv \psi$. Now, for every product in disjunctive normal form (DNF) of $\phi$, transformation $f_{1}$ constructs a graph with vertices $P_{+}$and an edge $\left(p_{1}, p_{2}\right)$ for every $\mathrm{d}\left(p_{1}\right)=\mathrm{d}\left(p_{2}\right)$ literal. Because $\mathrm{cp}\left(P, P_{+}\right) \equiv \psi$, if the resulting graph is connected, the product of the $\mathrm{d}\left(p_{1}\right)=\mathrm{d}\left(p_{2}\right)$ literals is equivalent to $\mathrm{Eq}(P)$. Thus, $f_{1}$ replaces every transition label $(\psi, \phi)$ in $\alpha$ with an equivalent label $\left(\psi, \phi^{\prime}\right)$, where $\phi^{\prime}$ denotes the modified DNF of $\phi$, with $\operatorname{Eq}(P)$ for every product of $\mathrm{d}\left(p_{1}\right)=\mathrm{d}\left(p_{2}\right)$ literals if those literals induce a connected graph. Let $\alpha^{\prime}$ denote the resulting CA. We can prove that $\alpha^{\prime} \sim \alpha$ holds (see Lemma 16 in [11, Appendix A]).

In the second step, transformation $f_{1}$ merges, for every pair of states $(q$, $q^{\prime}$ ), all transitions from $q$ to $q^{\prime}$ labeled by DC $\phi$ into one new transition. (The individual transitions differ only in their SC.) Every resulting transition has as its SC the sum of the SCs of the individual transitions. Figure 6 shows examples. We denote the resulting CA by $f_{1}(\alpha)$. The following proposition holds, because choices between individual transitions in $\alpha$ are encoded in $f_{1}(\alpha)$ by sum-scs of merged transitions. Consequently, $\alpha$ and $f_{1}(\alpha)$ can simulate each other's steps.

Proposition 1. $f_{1}(\alpha) \sim \alpha^{\prime}$ 


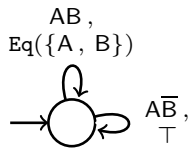

(a) LossySync

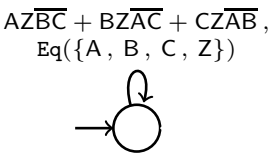

(b) Merger 3
$A H Z \overline{B Y}+A H Y \overline{B Z}+B H Z \overline{A Y}+B H Y \overline{A Z}$, $\mathrm{Eq}(\{\mathrm{A}, \mathrm{B}, \mathrm{H}, \mathrm{Y}, \mathrm{Z}\})$

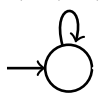

(c) Hourglass

Fig. 6. Application of transformations $f_{1}$ to the CA in Figure 2

\section{Transformation $f_{2}$ : Constructing Hypergraphs}

Every merged transition resulting from the previous preprocessing transformations can perhaps be implemented using queues along the same lines as transformation $h$ (see Section 3). In the first place, this depends on the extent to which ports in a merged transition are indistinguishable: no indistinguishable ports means no queues. Second, the SC of a merged transition should make port indistinguishability (i.e., queues), if present, apparent and mechanically detectable. The sCs of transitions in $f_{1}(\alpha)$ fail to do so. For instance, we (hence a computer) cannot directly derive from the syntax of $\mathrm{SC} A \mathrm{AZC}+\mathrm{BZ} \overline{\mathrm{AC}}+\mathrm{CZ} \overline{\mathrm{AB}}$ in Figure $6 \mathrm{~b}$ that its transition has a scalable implementation with queues. In contrast, the equivalent $\mathrm{SC} \bigoplus(\{\mathrm{A}, \mathrm{B}, \mathrm{C}\}) \cdot \mathrm{Z}$ makes this much more apparent. From this $\mathrm{SC}$, we can "obviously" (and mechanically by transformation $g_{2}$ in Figure 4c) conclude that ports $A, B$, and C may share the same queue, from which exactly one element is dequeued per firing, because they are indistinguishable indeed: intuitively, if $\delta \stackrel{s c}{=} \bigoplus(\{A, B, C\}) \cdot Z$, we cannot know which one of $A, B$, or C holds, unless we inspect $\delta$. Thus, beside automatically detecting indistinguishable ports in a transition, to actually reveal them as queues, we additionally need an algorithm for syntactically manipulating that transition's sc. We formulate both these aspects in terms of a per-transition hypergraph [13. Working with hypergraph representations simplifies our reasoning about, and manipulation of, sCs modulo associativity and commutativity. We compute hypergraphs as follows.

Let $\alpha=(Q, P, \longrightarrow, \imath)$ be an original $\mathrm{CA}$ as before, and let $\left(q, \psi, \phi, q^{\prime}\right)$ be a (merged) transition in $f_{1}(\alpha)$. Because $\alpha$ is an original CA and by the construction of $f_{1}(\alpha)$, we know that $\psi$ is a sum of $P$-complete products of ports (e.g., Figure 6). Because every single port $p$ is equivalent to $\bigoplus(\{p\})$, transformation $f_{2}$ can represent $\psi$ as a set $\mathcal{E}$ of sets $E$ of sets $V: \mathcal{E}$ represents the outer sum, every $E$ represents a $P$-complete product ( $E$ includes/excludes every positive/negative port), and every $V$ represents an inner exclusive sum. For instance, $\{\{\{A\},\{$ $Z\}\},\{\{B\},\{Z\}\},\{\{C\},\{Z\}\}\}$ represents the sc of the transition in Figure $6 \mathrm{~b}$. Transformation $f_{2}$ considers $\mathcal{E}$ as the set of hyperedges of a hypergraph over the set of vertices $\wp(\operatorname{Port}(\psi))$, where Port $(\psi)$ denotes the ports occurring in $\psi$ (i.e., every vertex is a set of ports). Formally, $f_{2}$ computes a function graph. Let $\mathbb{G R A P H}$ denote the set of all hypergraphs with sets of ports as vertices. 
Definition 1. graph $: \mathbb{S C} \rightarrow \mathbb{G R A P H}$ denotes the partial function from $\mathrm{SC} s$ to hypergraphs defined as 7

$$
\begin{aligned}
& \operatorname{graph}(\psi)=\left(\wp(\operatorname{Port}(\psi)),\left\{\begin{array}{l|l}
E & \begin{array}{l}
E=\left\{V \mid V=\{p\} \text { and } p \in P_{+}\right. \\
\text {and } P_{+} \subseteq \operatorname{Port}(\psi) \text { and } P_{+} \in \mathcal{P}
\end{array}
\end{array}\right\}\right) \\
& \text { if }\left[\psi=\sum\left(\left\{\begin{array}{ll}
\psi^{\prime} & \begin{array}{l}
\psi^{\prime} \equiv_{\mathrm{sc}} \operatorname{cp}\left(\operatorname{Port}(\psi), P_{+}\right) \text {and } \\
P_{+} \subseteq \operatorname{Port}(\psi) \text { and } P_{+} \in \mathcal{P}
\end{array}
\end{array}\right\}\right) \text { for some } \mathcal{P}\right]
\end{aligned}
$$

(The side condition states just that $\psi$ is a sum of $P$-complete products of ports.)

Figure 7 shows example hypergraphs (without unconnected vertices).

We define the meaning of a hypergraph as a sum of products of exclusive sums, where every product corresponds to a hyperedge. Such a product consists of exclusive sums of positive ports (one

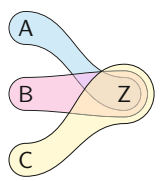

(a) Merger 3

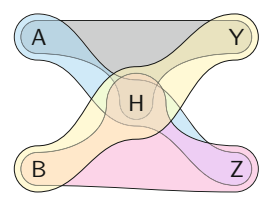

(b) Hourglass

Fig. 7. Hypergraphs for the transitions of the CA in Figure 6 for each vertex in the hyperedge), and it consists of negative ports (one for every port outside the vertices in the hyperedge). We can show that graph is an isomorphism (i.e., $\operatorname{graph}(\psi)$ is a sound and complete representation of $\psi$ ).

Definition 2. $\llbracket \cdot \rrbracket:(\wp(\mathbb{V E R}) \times \wp(\mathbb{P O R T})) \cup \mathbb{G R A P H} \rightarrow \mathbb{S} \mathbb{C}$ denotes the function from [hyperedge, set of ports]-pairs and hypergraphs to $\mathrm{SC} s$ defined as:

$$
\begin{array}{r}
\llbracket E \rrbracket_{P}=\prod(\{\psi \mid \psi=\bigoplus(V) \text { and } V \in E\} \cup \\
\{\psi \mid \psi=\bar{p} \text { and } p \in P \backslash(\bigcup E)\}) \\
\llbracket(\mathcal{V}, \mathcal{E}) \rrbracket=\sum(\{\psi \mid \psi=\llbracket E \rrbracket \cup \mathcal{V} \text { and } E \in \mathcal{E}\})
\end{array}
$$

Theorem 1. (Theorem 3 in [11, Appendix A])

$$
\psi \equiv \llbracket \operatorname{graph}(\psi) \rrbracket
$$

In summary, transformation $f_{2}$ computes graph for every merged transition in $f_{1}(\alpha)$ and stores each of those graphs in a set $\Gamma$ (indexed by transitions).

Hypergraphs as introduced are generic representations of synchronization patterns, isomorphic to but independent of SCs in CA. This reinforces that our optimization approach, transformation $f$, is not tied CA but a generally applicable technique when relevant context information is available.

\section{Transformation $f_{3}$ : Manipulating SCs}

Transformation $f_{3}$ aims at making all indistinguishable ports (hence queues) in SCs on (merged) transitions in $f_{1}(\alpha)$ apparent by analyzing and manipulating the hypergraphs in $\Gamma$, computed by transformation $f_{2}$. It consists of two steps.

In the first step, transformation $f_{3}$ computes the indistinguishable ports under every transition $t=\left(q, \psi, \phi, q^{\prime}\right)$ in $f_{1}(\alpha)$. We call the ports in a set $I$ indistinguishable under $t$ if for every distribution $\delta$ such that $\delta \stackrel{\text { sc }}{=} \psi$ and $\mid I \cap \operatorname{Dom}($

\footnotetext{
${ }^{7}$ Let $\wp(X)$ denote the power set of $X$.
} 
$\delta) \mid=1$, we cannot deduce from $\left.\delta\right|_{P \backslash I}$ which particular port in $I$ is satisfied by $\delta$. An example appeared in the first paragraph of Section 5 . In an implementation with a queue shared among the ports in $I$, this means that whenever $t$ fires, we know that exactly one port in $I$ participated in the transition but not which one, even if we know all other participating ports (i.e., those outside $I$ ).

By analyzing hypergraph $\gamma_{t} \in \Gamma$ for the SC $\psi$ of $t$, transformation $f_{3}$ computes maximal sets of indistinguishable ports under $t$ (larger sets of indistinguishable ports means larger queues means better scalability), as follows. Recall from Section 5 that $\gamma_{t}$ represents a sum (hyperedge relation) of $P$-complete products (hyperedges) of singleton exclusive sums (vertices). To understand how port indistinguishability displays in $\gamma_{t}$, suppose that ports $p_{1}, p_{2} \in P$ are indistinguishable, and let $\delta$ be a distribution such that $\delta \stackrel{\text { sc }}{=} \llbracket \gamma_{t} \rrbracket$. Because $\gamma_{t}$ 's hyperedge relation $\mathcal{E}$ represents a sum of $P$-complete products, exactly one hyperedge $E \in \mathcal{E}$ exists such that $\delta$ satisfies $\llbracket E \rrbracket_{P}$. Then, because $\left|\left\{p_{1}, p_{2}\right\} \cap \operatorname{Dom}(\delta)\right|=1$, a vertex $V \in E$ exists such that $p_{1} \in V$ or $p_{2} \in V \nabla$ In fact, because every hyperedge consists of singleton vertices, either $\left\{p_{1}\right\} \in E$ or $\left\{p_{2}\right\} \in E$. Now, by inspecting $\left.\delta\right|_{P \backslash\left\{p_{1}, p_{2}\right\}}$, we can infer the other vertices in $E$, beside either $\left\{p_{1}\right\}$ or $\left\{p_{2}\right\}$. Let $E^{\prime}$ denote this set of vertices, and observe that either $E=E_{1}=E^{\prime} \uplus\left\{\left\{p_{1}\right\}\right\}$ or $E=E_{2}=E^{\prime} \uplus\left\{\left\{p_{2}\right\}\right\}$. Because both options are possible, $\mathcal{E}$ necessarily includes both $E_{1}$ and $E_{2}$, and importantly, $E_{1}$ and $E_{2}$ are equal up to $p_{1}$ and $p_{2}$.

Generalizing this example from $\left\{p_{1}, p_{2}\right\}$ to arbitrarily sized sets $I$, informally, the ports in $I$ are indistinguishable if every port in $I$ is involved in the same hyperedges as every other port in I up to occurrences of ports in $I$. The following definitions make this generalization formally precise. First, we introduce a function Edge that determines for a port $p$ which hyperedges in $\mathcal{E}$ include $p$. (In fact, $\operatorname{Edge}(p, \mathcal{E})$ contains all such hypergedges up to occurrences of vertices with $p$.) Then, we define a function $\star$ that computes maximal sets of ports with the same set $\operatorname{Edge}(p, \mathcal{E})$. Importantly, $\star$ yields a partition of the set of ports in vertices connected by $\mathcal{E}$, denoted by $\operatorname{Port}(\mathcal{E})$. Henceforth, we therefore call every maximal set of indistinguishable ports computed by $\star$ a part.

Definition 3. Edge : PORT $\times \wp^{2}(\mathbb{V E R}) \rightarrow \wp^{2}(\mathbb{V E R})$ denotes the function from [port, set of hyperedges]-pairs to sets of hyperedges defined as:

$$
\operatorname{Edge}(p, \mathcal{E})=\{\mathcal{W} \mid \mathcal{W}=E \backslash\{V\} \text { and } p \in V \in E \in \mathcal{E}\}
$$

Definition 4. $\star: \wp^{2}(\mathbb{V E R}) \rightarrow \wp^{2}(\mathbb{P O R T})$ denotes the function from sets of hyperedges to sets of sets of ports defined as:

$$
\star(\mathcal{E})=\left\{P \mid P \in \wp^{+}(\operatorname{Port}(\mathcal{E})) \text { and }[[p \in P \text { iff } \mathcal{T}=\operatorname{Edge}(p, \mathcal{E})] \text { for all } p]\right\}
$$

Lemma 1. (Lemma 12 in [11, Appendix A])

1. $\bigcup \star(\mathcal{E})=\operatorname{Port}(\mathcal{E})$

2. $\left[P_{1} \neq P_{2}\right.$ and $\left.P_{1}, P_{2} \in \star(\mathcal{E})\right]$ implies $P_{1} \cap P_{2}=\emptyset$

\footnotetext{
${ }^{8}$ Otherwise, if $p_{1}, p_{2} \notin V$ for all $V \in E$, the $P$-complete product represented by $E$ contains $\bar{p}_{1}$ and $\bar{p}_{2}$ such that $\delta \notin \neq p_{1}$ and $\delta \notin p_{2}$. This contradicts the assumption $\left|\left\{p_{1}, p_{2}\right\} \cap \operatorname{Dom}(\delta)\right|=1$, which implies either $\delta \stackrel{\text { sc }}{=} p_{1}$ or $\delta \stackrel{\text { sc }}{=} p_{2}$.
} 


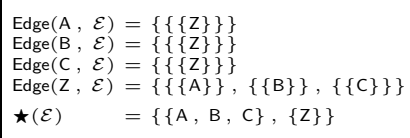

(a) Merger $_{3}$

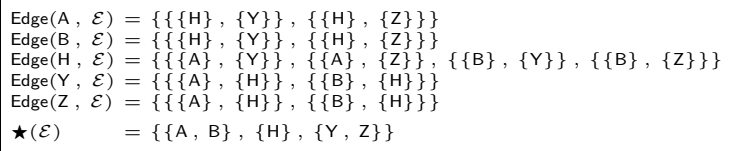

(b) Hourglass

Fig. 8. Maximal sets of indistinguishable ports of the hypergraphs in Figure 7

In summary, in the first step, transformation $f_{3}$ computes maximal sets of indistinguishable ports in every merged transition $t=\left(q, \psi, \phi, q^{\prime}\right)$ by applying $\star$ to hyperedge relation $\mathcal{E}$ in hypergraph $\gamma_{t}$ for $\psi$. Figure 8 shows examples.

In the second step, $f_{3}$ manipulates $\mathcal{E}$ of every hypergraph $\gamma_{t}$ such that afterward, every vertex in every hyperedge in $\mathcal{E}$ is a part in $\star(\mathcal{E})$. Importantly, every vertex $V \in E \in \mathcal{E}$ such that $V \in \star(\mathcal{E})$ represents not just any $\bigoplus$-formula but one of indistinguishable ports. Consequently, in the meaning of the manipulated $\gamma_{t}$, indistinguishable ports become apparent as inner $\bigoplus$-formulas as in the example in the first paragraph of Section 5.

For manipulating hyperedge relation $\mathcal{E}$, we introduce an operation $\sqcup$ that combines two combinable hyperedges into one in a semantics-preserving way. Roughly, we call two distinct hyperedges $E_{1}, E_{2} \in \mathcal{E}$ combinable if we can select disjoint vertices $V_{1}, V_{2} \in E_{1} \cup E_{2}$ such that $E_{1}$ and $E_{2}$ are equal up to inclusion of $V_{1}$ and $V_{2}$. We denote this property as $\left(E_{1}, V_{1}\right) \curlyvee_{\mathcal{E}}\left(E_{2}, V_{2}\right)$. Applied to combinable hyperedges $E_{1}$ and $E_{2}$, operation $\sqcup$ removes $E_{1}$ and $E_{2}$ from $\mathcal{E}$ and adds their combination $E_{\dagger}=\left\{V_{1} \cup V_{2}\right\} \cup\left(E_{1} \cap E_{2}\right)$ to $\mathcal{E}$. Formally, we have the following. Let $\mathbb{V E R}$ denote the set of all vertices.

Definition 5. $\curlyvee \subseteq(\wp(\mathbb{V E R}) \times \mathbb{V} E R) \times(\wp(\mathbb{V E R}) \times \mathbb{V} E R) \times \wp^{2}(\mathbb{V E R})$ denotes the relation on tuples consisting of two sets of [hyperedge, vertex]-pairs and a set of hyperedges defined as:

$$
\left(E_{1}, V_{1}\right) \curlyvee_{\mathcal{E}}\left(E_{2}, V_{2}\right) \text { iff }\left[\begin{array}{c}
E_{1}, E_{2} \in \mathcal{E} \text { and } E_{1} \neq E_{2} \text { and } V_{1} \cap V_{2}=\emptyset \\
\text { and } E_{1}=\left(E_{2} \backslash\left\{V_{2}\right\}\right) \cup\left\{V_{1}\right\} \\
\text { and } E_{2}=\left(E_{1} \backslash\left\{V_{1}\right\}\right) \cup\left\{V_{2}\right\}
\end{array}\right]
$$

Definition 6. $\sqcup:(\wp(\mathbb{V E R}) \times \mathbb{V E R}) \times(\wp(\mathbb{V E R}) \times \mathbb{V}$ ER $) \times \wp^{2}(\mathbb{V E R}) \rightarrow \wp^{2}(\mathbb{V E R})$ denotes the partial function from tuples consisting of two [hyperedge, vertex]pairs and a set of hyperedges to sets of hyperedges defined as:

$$
\begin{gathered}
\left.\left(E_{1}, V_{1}\right) \sqcup_{\mathcal{E}}\left(E_{2}, V_{2}\right)=\mathcal{E} \backslash\left\{E_{1}, E_{2}\right\}\right) \cup\left\{\left\{V_{1} \cup V_{2}\right\} \cup\left(E_{1} \cap E_{2}\right)\right\} \\
\text { if }\left(E_{1}, V_{1}\right) \curlyvee_{\mathcal{E}}\left(E_{2}, V_{2}\right)
\end{gathered}
$$

Lemma 2. (Lemma 8 in [11, Appendix A])

$$
\left(E_{1}, V_{1}\right) \curlyvee_{\mathcal{E}}\left(E_{2}, V_{2}\right) \text { implies } \llbracket(\mathcal{V}, \mathcal{E}) \rrbracket \equiv_{\mathrm{sc}} \llbracket\left(\mathcal{V},\left(E_{1}, V_{1}\right) \sqcup_{\mathcal{E}}\left(E_{2}, V_{2}\right)\right) \rrbracket
$$

Transformation $f_{3}$ uses operation $\sqcup$ in the algorithm for combining hyperedges in Figure 9. Essentially, as long as vertices $V_{1}$ and $V_{2}$ exist such that the ports 


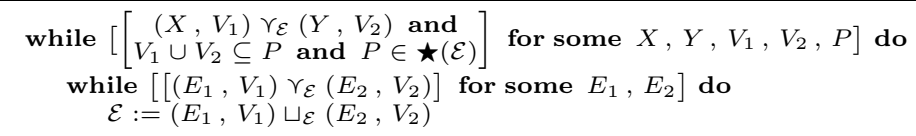

Fig. 9. Algorithm for combining hyperedges

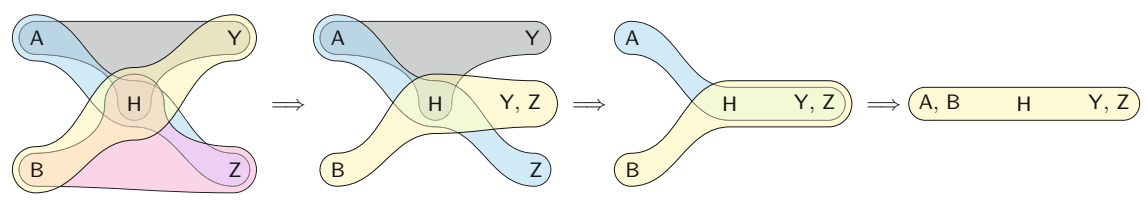

Fig. 10. Evolution of the hypergraphs in Figure $7 \mathrm{~b}$

in $V_{1} \cup V_{2}$ are indistinguishable (as computed by $\star$ ), the algorithm combines all combinable hyperedges that include $V_{1}$ and $V_{2}$. For instance, Figure 10 shows the evolution of the hypergraph in Figure $7 \mathrm{~b}$ during the run of the algorithm in which it first selects $\mathrm{Y}$ and $\mathrm{Z}$ as $V_{1}$ and $V_{2}$ and afterward $\mathrm{A}$ and B. (In another run, the algorithm may change this order to obtain the same result.)

Let $\mathcal{E}_{\text {in }}$ and $\mathcal{E}_{\text {out }}$ denote the sets of hyperedges before and after running the algorithm. To consider the algorithm correct, $\mathcal{E}_{\text {out }}$ must satisfy two properties: it should represent an sc equivalent to the sc represented by $\mathcal{E}_{\text {in }}$ (i.e., the algorithm is semantics-preserving), and every vertex in every hyperedge in $\mathcal{E}_{\text {out }}$ should be a part in $\star\left(\mathcal{E}_{\text {in }}\right)$ (i.e., the algorithm effectively reveals indistinguishability). We use Hoare logic to prove these properties 14,15. In particular, we can show that the triple $\{$ Pre $\} A$ Post $\}$ holds, where $A$ denotes the algorithm in Figure 9 Precondition Pre states that $\gamma_{t}=\left(\mathcal{V}, \mathcal{E}_{\text {in }}\right)$ is a hypergraph (for the sC of transition $t$ ) such that every port in a connected vertex inhabits at most one connected vertex, and such that every connected vertex is nonempty. The definition of graph in Definition 1 implies these conditions. (However, because its precondition is more liberal, the algorithm is more generally applicable.) The postcondition Post states that correctness as previously formulated holds. Formally:

$$
\left.\llbracket\left(\mathcal{V}, \mathcal{E}_{\text {out }}\right) \rrbracket=\llbracket\left(\mathcal{V}, \mathcal{E}_{\text {in }}\right) \rrbracket \text { and }\left[\begin{array}{c}
E \in \mathcal{E}_{\text {out }} \text { implies } \\
E \subseteq \star\left(\mathcal{E}_{\text {in }}\right)
\end{array}\right] \text { for all } E\right]
$$

Figure 11 shows the algorithm annotated with assertions for total correctness. By the axioms and rules of Hoare logic, this proof is valid if we can prove that for all six pairs of consecutive assertions, the upper assertion implies the lower one. For brevity, below, we discuss some salient aspects.

First, the algorithm terminates, because (i) every iteration of the outer loop consists of at least one iteration of the inner loop, for $X=E_{1}$ and $Y=E_{2}$, (ii) in every iteration of the inner loop, $\mathcal{E}$ decreases by one, and (iii) $\mathcal{E}$ is finite. Second, the algorithm is semantics-preserving by Lemma 2 The main challenge is proving that the algorithm is also effective. A notable step in this proof is establishing the property labeled Interm from $\operatorname{lnv}_{2}$ (the invariant of the inner 


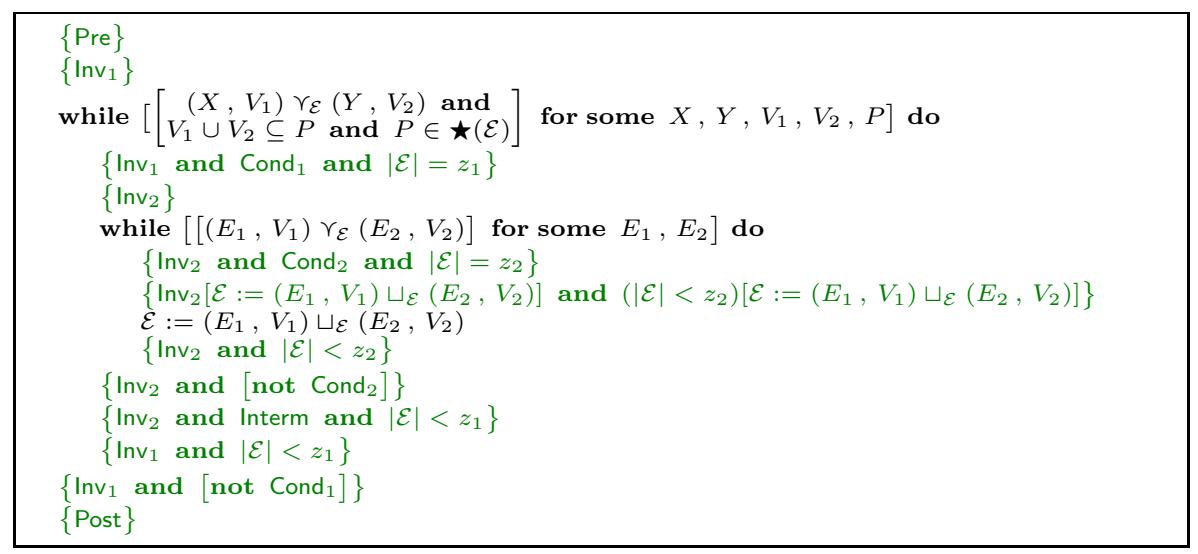

Fig. 11. Algorithm for combining hyperedges with assertions for total correctness

loop) and [not Cond 2 (the negation of the inner loop's condition). Informally, Interm states that if $\mathcal{F}$ denotes the hyperedge relation before running the inner loop, we have $\mathcal{E}=\mathcal{F} \backslash\left(\mathcal{F}_{1,2}\right) \cup \mathcal{F}_{\dagger}$ after running the inner loop. Here, $\mathcal{F}_{1,2}$ contains all hyperedges from $\mathcal{F}$ that include $V_{1}$ or $V_{2}$, while $\mathcal{F}_{\dagger}$ denotes all new hyperedges added by $\sqcup$ during the loop. This property subsequently enables us to prove $\operatorname{lnv}_{1}$ (the invariant of the outer loop), which among other properties states $\star\left(\mathcal{E}_{\text {in }}\right)=\star(\mathcal{E})$. Consequently, to prove the algorithm's effectiveness, it suffices to show that $E \in \mathcal{E}_{\text {out }}$ implies $E \subseteq \star\left(\mathcal{E}_{\text {out }}\right)$ (for all $E$ ).

Theorem 2. (Theorem 4 in [11, Appendix A]) $\{$ Pre $\}$ A Post

In summary, in the second step, for every (merged) transition $t=(q, \psi, \phi$, $q^{\prime}$ ) in $f_{1}(\alpha)$, transformation $f_{3}$ manipulates hypergraph $\gamma_{t}$ to $\gamma_{t}^{\prime}$ by running the algorithm in Figure 9, given the maximal sets of indistinguishable ports computed in $f_{3}$ 's first step with $\star$. Afterward, $f_{3}$ replaces $\psi$ in $t$ with $\llbracket \gamma_{t}^{\prime} \rrbracket$, which by the correctness of the algorithm is equivalent to $\llbracket \gamma_{t} \rrbracket$ and has made indistinguishable ports (hence queues) apparent. We denote the resulting transition relation by $\left(f_{3} \circ f_{1}\right)(\longrightarrow)$ and the resulting CA by $\left(f_{3} \circ f_{1}\right)(\alpha)$. Because $\psi \equiv_{\text {sc }} \llbracket \gamma_{t} \rrbracket \equiv_{\text {sc }} \llbracket \gamma_{t}^{\prime} \rrbracket$ for all transitions $t$ in $f_{1}(\alpha)$, the following proposition follows from Lemma 16 in [11, Appendix A]. Together, Propositions 1 and 2 imply that transformation $f$ is semantics-preserving.

Proposition 2. $\left(f_{3} \circ f_{1}\right)(\alpha) \sim f_{1}(\alpha)$

We end with some examples in Figure 12. Transformation $f_{3}$ has not had any effect on the LossySync CA, so its implementation does not benefit from queues (no indistinguishable ports), as expected. The Merger 3 and Hourglass $\mathrm{CA}$, in contrast, have changed significantly. In the SC of Merger $_{3}$, we can now clearly recognize one queue for ports $A, B$, and $C$ and one queue for port Z (cf. transformation $h$ in Section [3); similarly, in the SC of Hourglass, we can now clearly recognize one queue for ports $A$ and $B$ and one queue for ports $Y$ and $Z$. 


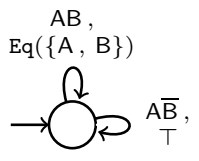

(a) LossySync

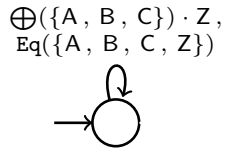

(b) Merger $_{3}$
$\bigoplus(\{A, B\}) \cdot H \cdot \bigoplus(\{Y, Z\})$,

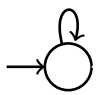

(c) Hourglass

Fig. 12. Application of transformation $f_{3}$ to the CA in Figure 6

Applied to Merger $_{3}$, transformation $f$ optimizes a multiple-producer-singleconsumer protocol. More abstractly, in this case, $f$ optimizes a protocol among two groups of processes, $X_{1}$ (producers) and $X_{2}$ (consumer), such that $\left|X_{1}\right|=3$ and $\left|X_{2}\right|=1$ and all processes in $X_{1}$ are indistinguishable to all processes in $X_{2}$ and vice versa. Generally, $f$ can optimize protocols among $n$ groups of processes $X_{1}, \ldots, X_{n}$ such that for all $1 \leq i, j \leq n$, all processes in $X_{i}$ are indistinguishable to all processes in $X_{j}$ and vice versa. For instance, applied to Hourglass, $f$ optimizes a protocol among three groups of processes such that $\left|X_{1}\right|=\left|X_{3}\right|=2$ and $\left|X_{2}\right|=1$.

After having applied transformation $f$, the automatic generation of actual implementations is straightforward (i.e., transformation $g_{2}$ in Figure 4c). The resulting code is, in fact, exactly the same as the code that results from manually applying transformation $h$ as in Section 3 (and consequently, it has the same performance): instead of checking an event structure for every port as preoptimized code does, optimized code checks one eventQueue structure for every maximal set of indistinguishable ports, which transformation $f$ has made explicit as $\bigoplus$-formulas in SCs (and are thus easy to detect in the $f$-transformed CA). As such, optimized code checks the SC of all transitions in the pre-transformation CA that differ only in indistinguishable ports (before applying $f$ ) at the same time. For $k$ such transitions, consequently, an unscalable exhaustive $\mathcal{O}(k)$ search is optimized to perfectly scalable $\mathcal{O}(1)$ queue operations. Thus, with respect to Figure $4 \mathrm{c}$, the fully mechanical transformation $g_{2} \circ f=g_{2} \circ f_{3} \circ f_{2} \circ f_{1}$ yields the same code and scalability as the partially manual transformation $h \circ g_{1}$.

\section{Concluding Remarks}

In this paper, we analyzed scalability issues of the code generated by our Reoto- $\mathrm{C}$ compiler, we explained a manual solution, and we studied the various steps of a mechanical procedure for transforming a CA $\alpha$ to an equivalent CA $\beta$, which makes port indistinguishability (hence queues) maximally apparent, using the $\bigoplus$-operator. Our tool can use this mechanical procedure to generate code for $\alpha$ via $\beta$ with good scalability. In particular, whereas unoptimized code generated for $\alpha$ requires $\mathcal{O}(k)$ time to compute eligibility of $k$ transitions - essentially an exhaustive search - the optimized code generated for $\beta$ requires only $\mathcal{O}(1)$ time: all maximal sets of indistinguishable ports (explicit in $\beta$ as a $\bigoplus$-formulas in sCs) in the implementation share the same queue, which optimizes the unscalable $\mathcal{O}(k)$ search to perfectly scalable $\mathcal{O}(1)$ queue operations. 
Although inspired by our work on a Reo compiler and formulated generally in terms of $\mathrm{CA}$, we make contributions beyond Reo and CA. The synchronization pattern that we identified and optimized is common and occurs in many classes of protocols and their implementation, regardless of the particular language. Therefore, compilers for other high-level languages may use the same approach as explained in this paper to similarly optimize code generated for programs in those languages. In fact, this paper led to adding new features to Proto-Runtime to enable our optimization technique, thereby facilitating efficient implementation of our $f$-transformed CA. Importantly, these new features in Proto-Runtime can now benefit other languages implemented on top of Proto-Runtime as well.

Automatically performing our optimization directly on low-level code such as $\mathrm{C}$ (instead of on $\mathrm{CA}$ ) is extremely complex, if not impossible. This shows that using higher-level languages (that preserve relevant context information about protocols) for multicore programming can indeed be advantageous for performance, a significant general observation in language and compiler design for multicore platforms. Indeed, the work presented in this paper serves as evidence that it is possible not only to specify interaction protocols at a higher level of abstraction (than locks, mutex, semaphores, message exchanges, etc.) but also automatically compile and optimize such high-level specifications down to executable code. Such higher-level specifications convey more of the intention behind the protocol, which gives more room for a compiler/optimizer to find and apply efficient implementation alternatives. Lower-level, more imperative, specifications of interaction protocols either lose or obscure the intentions behind protocols and seriously constrict the ability of compilers/optimizers to find efficient implementation alternatives. See [1] for related work on high-level approaches to multicore programming.

This paper makes primarily conceptual and theoretical contributions, and we used performance figures only to motivate and explain the development of our optimization technique. An in-depth study of the use of this technique in practice, including more benchmarks and experiments with different kinds of protocols and contexts, is our next objective, now that we know that the technique is correct. As part of this future work, we will also extend our current, limited proof-of-concept implementation (used in obtaining the data for Figure 5) to a full implementation. We end with the following remarks.

Indistinguishability of data. Transformation $f$ effectively merges transitions with labels of the form $(\psi, \mathrm{Eq}(P))$. The reason is that the ports $\mathrm{in} \operatorname{Eq}(P)$ are indistinguishable from a data perspective. (Whether those ports are also indistinguishable in $\psi$ is exactly what transformation $f_{3}$ investigates.) Detecting port indistinguishability in arbitrary DCs so as to improve the applicability of $f$ seems an interesting and important future challenge.

Guarded automata. Our scs, as arbitrary propositional formulas, seem similar to guards on transitions in the guarded automata used by Bonsangue et al. for modeling connector behavior [16]. The intuitive meaning of such guards, however, significantly differs: guards specify a constraint on the environment, 
while sCs specify a constraint on an execution step. (In fact, transition labels of guarded automata carry both a guard and an SC.)

Model-based testing. We skipped an explanation of the actual code generation process (i.e., transformation $g_{2}$ in Figure 4), dismissing it as "straightforward" and "obviously correct". An interesting line of work to better substantiate the latter statement is to have our tool generate not only executable code but also test cases derived from the input CA. Kokash et al. have already worked on such model-based testing for $\mathrm{CA}$ in a different context [17].

\section{References}

1. Arbab, F.: Reo: a channel-based coordination model for component composition. MSCS 14(3), 329-366 (2004)

2. Arbab, F.: Puff, The Magic Protocol. In: Agha, G., Danvy, O., Meseguer, J. (eds.) Talcott Festschrift. LNCS, vol. 7000, pp. 169-206. Springer, Heidelberg (2011)

3. Jongmans, S.S., Arbab, F.: Modularizing and Specifying Protocols among Threads. In: Proceedings of PLACES 2012. EPTCS. CoRR, vol. 109, pp. 34-45 (2013)

4. Jongmans, S.S., Arbab, F.: Overview of Thirty Semantic Formalisms for Reo. SACS 22(1), 201-251 (2012)

5. Kokash, N., Krause, C., de Vink, E.: Reo+mCRL2: A framework for modelchecking dataflow in service compositions. FAC 24(2), 187-216 (2012)

6. Jongmans, S.S., Halle, S., Arbab, F.: Reo: A Dataflow Inspired Language for Multicore. In: Proceedings of DFM 2013 (2013)

7. Baier, C., Sirjani, M., Arbab, F., Rutten, J.: Modeling component connectors in Reo by constraint automata. SCP 61(2), 75-113 (2006)

8. Halle, S.: A Study of Frameworks for Collectively Meeting the Productivity, Portability, and Adoptability Goals for Parallel Software. PhD thesis, University of California, Santa Cruz (2011)

9. Halle, S., Cohen, A.: A Mutable Hardware Abstraction to Replace Threads. In: Rajopadhye, S., Mills Strout, M. (eds.) LCPC 2011. LNCS, vol. 7146, pp. 185-202. Springer, Heidelberg (2013)

10. Butenhof, D.: Programming with POSIX Threads. Addison-Wesley (1997)

11. Jongmans, S.S., Halle, S., Arbab, F.: Automata-based Optimization of Interaction Protocols for Scalable Multicore Platforms (Technical Report). Technical Report FM-1402, CWI (2014)

12. Sirjani, M., Jaghoori, M.M., Baier, C., Arbab, F.: Compositional Semantics of an Actor-Based Language Using Constraint Automata. In: Ciancarini, P., Wiklicky, H. (eds.) COORDINATION 2006. LNCS, vol. 4038, pp. 281-297. Springer, Heidelberg (2006)

13. Bretto, A.: Hypergraph Theory: An Introduction. Springer (2013)

14. Hoare, T.: An Axiomatic Basis for Computer Programming. CACM 12(10), 576-580 (1969)

15. Apt, K., de Boer, F., Olderog, E.R.: Verification of Sequential and Concurrent Programs. Springer (2009)

16. Bonsangue, M., Clarke, D., Silva, A.: A model of context-dependent component connectors. SCP 77(6), 685-706 (2009)

17. Kokash, N., Arbab, F., Changizi, B., Makhnist, L.: Input-output Conformance Testing for Channel-based Service Connectors. In: Proceedings of PACO 2011. EPTCS. CoRR, vol. 60, pp. 19-35 (2011) 